\title{
Rational Catalan Combinatorics: The Associahedron
}

\section{Drew Armstrong $\|^{\mid} \quad$ Brendon Rhoades $\|^{2} \quad$ Nathan Williams ${ }^{3}$}

${ }^{1}$ Department of Mathematics, University of Miami, USA

${ }^{2}$ Department of Mathematics, University of California - San Diego, USA

${ }^{3}$ Department of Mathematics, University of Minnesota, USA

Abstract. Each positive rational number $x>0$ can be written uniquely as $x=a /(b-a)$ for coprime positive integers $0<a<b$. We will identify $x$ with the pair $(a, b)$. In this extended abstract we use rational Dyck paths to define for each positive rational $x>0$ a simplicial complex $\operatorname{Ass}(x)=\operatorname{Ass}(a, b)$ called the rational associahedron. It is a pure simplicial complex of dimension $a-2$, and its maximal faces are counted by the rational Catalan number

$$
\operatorname{Cat}(x)=\operatorname{Cat}(a, b):=\frac{(a+b-1) !}{a ! b !} .
$$

The cases $(a, b)=(n, n+1)$ and $(a, b)=(n, k n+1)$ recover the classical associahedron and its Fuss-Catalan generalization studied by Athanasiadis-Tzanaki and Fomin-Reading. We prove that Ass $(a, b)$ is shellable and give nice product formulas for its $h$-vector (the rational Narayana numbers) and $f$-vector (the rational Kirkman numbers). We define $\operatorname{Ass}(a, b)$.

Résumé. Tout nombre rationnel positif $x>0$ peut être exprimé de façon unique par $x=a /(b-a)$ avec $0<a<b$ deux entiers positifs premiers entre eux. Nous identifierons $x$ avec la paire $(a, b)$. Dans cet article, nous utilisons les chemins de Dyck rationnels pour définir pour tout rationnel positif $x>0$ un complexe $\operatorname{simplicial~} \operatorname{Ass}(x)=\operatorname{Ass}(a, b)$ que nous appelons l'associahedron rationnel. Il s'agit d'un complexe simplicial pur de dimension $a-2$, et ses faces maximales sont comptées par le nombre rationnel de Catalan

$$
\operatorname{Cat}(x)=\operatorname{Cat}(a, b):=\frac{(a+b-1) !}{a ! b !} .
$$

Les cas $(a, b)=(n, n+1)$ et $(a, b)=(n, k n+1)$ permettent de retrouver l'associhedron classique et sa généralisation Fuss-Catalan, étudiée par Athanasiadis-Tzanaki et Fomin-Reading. Nous démontrons que Ass $(a, b)$ est shellable et nous donnons des formules de produits simples pour son $h$-vecteur (les nombres rationnels de Narayana) et son $f$-vecteur (les nombres rationnels de Kirkman).

Keywords: associahedron, Dyck path, $f$-vector, $h$-vector, shelling, noncrossing partition

\footnotetext{
†Partially supported by NSF grant DMS-1001825

${ }_{\ddagger}$ Partially supported by NSF grant DMS-1068861

1365-8050 @ 2013 Discrete Mathematics and Theoretical Computer Science (DMTCS), Nancy, France
} 


\section{Motivation}

This extended abstract is one of a pair of papers (see also [ALW]) that initiate the research program of $r a-$ tional Catalan combinatorics. The motivation for this program is both combinatorial and representationtheoretic.

The classical Catalan number $\underbrace{(i)}$

$$
\text { Cat }(n, n+1)=\frac{1}{n+1}\left(\begin{array}{c}
2 n \\
n
\end{array}\right)
$$

are among the most important sequences in combinatorics. As of this writing, they are known to count at least 201 distinct families of combinatorial objects [Stan]. For our current purpose, the following three are the most important:

1. Dyck paths from $(0,0)$ to $(n, n)$,

2. Triangulations of a convex $(n+2)$-gon, and

3. Noncrossing partitions of a cycle $(1,2, \ldots, n)$.

There are two observations that have spurred recent progress in this field. The first is that Catalan objects are revealed to be type $A$ phenomena (corresponding to the symmetric group) when properly interpreted in the context of reflection groups. The second is that many definitions of Catalan objects can be further generalized to accommodate an additional parameter, so that the resulting objects are counted by Fuss-Catalan numbers (see [Arm, Chapter 5]).

Both of these generalizations can be motivated from Garsia's and Haiman's [GH] observation that the Catalan numbers play a deep role in representation theory. The symmetric group $\mathfrak{S}_{n}$ acts on the polynomial ring $D S_{n}:=\mathbb{Q}\left[x_{1}, \ldots, x_{n}, y_{1}, \ldots, y_{n}\right]$ by permuting variables diagonally. That is, for $w \in$ $\mathfrak{S}_{n}$ we define $w \cdot x_{i}=x_{w(i)}$ and $w \cdot y_{i}=y_{w(i)}$. Weyl [W] proved that the subring of diagonal invariants is generated by the polarized power sums $p_{r, s}=\sum_{i} x_{i}^{r} y_{i}^{s}$ for $r+s \geq 0$ with $1 \leq r+s \leq n$. The quotient ring of diagonal coinvariants $D R_{n}:=D S_{n} /\left(p_{r, s}\right)$ inherits the structure of an $\mathfrak{S}_{n}$-module which is bigraded by $x$-degree and $y$-degree. Garsia and Haiman conjectured that $\operatorname{dim} D R_{n}=(n+1)^{n-1}$ (a number famous from Cayley's formula [Cay] $)$ and that the dimension of the sign-isotypic component is the Catalan number Cat $(n, n+1)$. These conjectures turned out to be difficult to resolve, and were proved about ten years later by Haiman using the geometry of Hilbert schemes.

An excellent introduction to this subject is Haiman's paper [Hai1], in which he laid the foundation for generalizing the theory of diagonal coinvariants to other reflection groups. Let $W$ be a Weyl group, so that $W$ acts irreducibly on $\mathbb{R}^{\ell}$ by reflections and stabilizes a full-rank lattice $\mathbb{Z}^{\ell} \approx Q \subseteq \mathbb{R}^{\ell}$, called the root lattice. The group also comes equipped with special integers $d_{1} \leq \cdots \leq d_{\ell}$ called degrees, of which the largest $h:=d_{\ell}$ is called the Coxeter number. Haiman showed that number of orbits of $W$ acting on the finite torus $Q /(h+1) Q$ is equal to

$$
\operatorname{Cat}(W):=\prod_{i} \frac{h+d_{i}}{d_{i}},
$$

which we now refer to as the Catalan number of $W$.

From this modern perspective, our three examples above become:

(i) This notation will we justified shortly. 
1. $W$-orbits of the finite torus $Q /(h+1) Q$ [Shi1, Hai1, Ath1, CP],

2. Clusters in Fomin and Zelevinsky's finite type cluster algebras [FZ], and

3. Elements beneath a Coxeter element $c$ in the absolute order on $W$ [Rei, Arm].

More generally, given any positive integer $p$ coprime to the Coxeter number $h$, Haiman showed that the number of orbits of $W$ acting on the finite torus $Q / p Q$ is equal to

$$
\operatorname{Cat}(W, p):=\prod_{i} \frac{p+d_{i}-1}{d_{i}},
$$

which we now refer to as a rational Catalan number.

The cases $p=m h+1$ have been extensively studied as the Fuss-Catalan analogues, which further generalize our initial three examples to:

1. Dominant regions in the $m$-Shi arrangement [Ath2, FV],

2. Clusters in the generalized cluster complex [FR], and

3. $m$-multichains in the noncrossing partition lattice. [Edel, Arm].

The broad purpose of rational Catalan combinatorics is to complete the generalization from $p=+1 \bmod h$ to all parameters $p$. That is, we wish to define and study Catalan objects such as parking functions, Dyck paths, triangulations, and noncrossing partitions for each pair $(W, p)$, where $W$ is a finite reflection group and $p$ is a positive integer coprime to the Coxeter number $h$. We may think of this as a twodimensional problem with a "type axis" $W$ and a "parameter axis" $p$. The level set $p=h+1$ is understood fairly well, and the Fuss-Catalan cases $p=+1 \bmod h$ are discussed in Chapter 5 of Armstrong [Arm]. However, it is surprising that the type $A$ level set (i.e. $W=\mathfrak{S}_{n}$ ) is an open problem. This could have been pursued fifty years ago, but no one has done so in a systematic way.

Thus, we propose to begin the study of rational Catalan combinatorics with the study of classical rational Catalan combinatorics corresponding to a pair $\left(\mathfrak{S}_{a}, b\right)$ with $b$ coprime to $a$. In this case, we have the classical rational Catalan number

$$
\operatorname{Cat}\left(\mathfrak{S}_{a}, b\right)=\frac{1}{a+b}\left(\begin{array}{c}
a+b \\
a, b
\end{array}\right)=\frac{(a+b-1) !}{a ! b !} .
$$

Note the surprising symmetry between $a$ and $b$; i.e. that $\operatorname{Cat}\left(\mathfrak{S}_{a}, b\right)=\operatorname{Cat}\left(\mathfrak{S}_{b}, a\right)$. This will show up as a conjectural Alexander duality in our study of rational associahedra.

First we will set down notation for the rational Catalan numbers Cat $\left(\mathfrak{S}_{a}, b\right)$ in Section 2 Then in Section 3 we will define the rational Dyck paths which are the heart of the theory. In Section 4 we will use the Dyck paths to define and study rational associahedra. (In the full version of this paper we will also study the closely related rational noncrossing partitions.) In a separate paper [ALW] the Dyck paths will be used to define and study rational parking functions and $q, t$-statistics on these. The project of generalizing these constructions to reflection groups beyond $\mathfrak{S}_{n}$ is left for the future. 


\section{Rational Catalan Numbers}

Given a rational number $x \in \mathbb{Q}$ outside the range $[-1,0]$, there is a unique way to write $x=a /(b-a)$ where $a \neq b$ are coprime positive integers. We consider this a canonical form, and we will identify $x \in \mathbb{Q}$ with the ordered pair $(a, b) \in \mathbb{N}^{2}$ when convenient.

Inspired by the formulas (1) and (2) above, we define the rational Catalan number:

$$
\operatorname{Cat}(x)=\operatorname{Cat}(a, b):=\frac{1}{a+b}\left(\begin{array}{c}
a+b \\
a, b
\end{array}\right)=\frac{(a+b-1) !}{a ! b !} .
$$

The most important feature of the rational Catalan numbers is that they are backwards-compatible:

$$
\operatorname{Cat}(n)=\operatorname{Cat}(n / 1)=\operatorname{Cat}(n, n+1)=\frac{1}{2 n+1}\left(\begin{array}{c}
2 n+1 \\
n, n+1
\end{array}\right)=\frac{1}{n+1}\left(\begin{array}{c}
2 n \\
n
\end{array}\right) .
$$

But note also that Cat $(a, b)$ is symmetric in $a$ and $b$. This, together with the fact that $a /(b-a)=x$ if and only if $b /(a-b)=-x-1$, gives us

$$
\operatorname{Cat}(x)=\operatorname{Cat}(a, b)=\operatorname{Cat}(b, a)=\operatorname{Cat}(-x-1) .
$$

That is, the function Cat : $\mathbb{Q} \backslash[-1,0] \rightarrow \mathbb{N}$ is symmetric about $x=-1 / 2$. Now observe that $-\frac{1}{x-1}-1=$ $\frac{x}{1-x}$, and hence $\operatorname{Cat}(1 /(x-1))=\operatorname{Cat}(x /(1-x))$. We call this value the derived Catalan number:

$$
\operatorname{Cat}^{\prime}(x):=\operatorname{Cat}(1 /(x-1))=\operatorname{Cat}(x /(1-x)) .
$$

Furthermore, note that $\frac{1}{(1 / x)-1}=\frac{x}{1-x}$, hence

$$
\operatorname{Cat}^{\prime}(x)=\operatorname{Cat}^{\prime}(1 / x)
$$

We call this equation rational duality and it will play an important role in our study of rational associahedra. Equation (3) can also be used to extend the domain of Cat' from $\mathbb{Q} \backslash[-1,0]$ to $\mathbb{Q} \backslash\{0\}$, but we don't know if this holds combinatorial significance. In terms of $a$ and $b$ we can write

$$
\operatorname{Cat}^{\prime}(x)=\operatorname{Cat}^{\prime}(a, b)= \begin{cases}\left(\begin{array}{l}
b \\
a
\end{array}\right) / b & \text { if } a<b, \\
\left(\begin{array}{l}
a \\
b
\end{array}\right) / a & \text { if } b<a .\end{cases}
$$

The "derivation" of Catalan numbers can be viewed as a "categorification" of the Euclidean algorithm. For example, consider $x=5 / 3$ (that is, $a=5$ and $b=8$ ). The continued fraction expansion of $x$ is

$$
\frac{5}{3}=1+\frac{1}{1+\frac{1}{1+\frac{1}{1}}}
$$




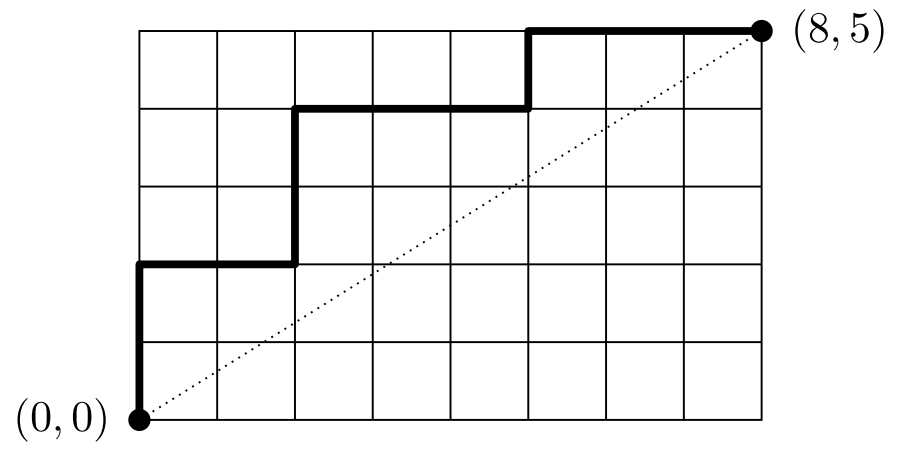

Fig. 1: This is a $(5,8)$-Dyck path.

with "convergents" (that is, successive truncations) $\frac{1}{1}, \frac{2}{1}, \frac{3}{2}, \frac{5}{3}$. Thus we have

$$
\begin{aligned}
\operatorname{Cat}(5 / 3) & =99, \\
\operatorname{Cat}^{\prime}(5 / 3) & =\operatorname{Cat}(3 / 2)=7, \\
\operatorname{Cat}^{\prime \prime}(5 / 3) & =\operatorname{Cat}^{\prime}(3 / 2)=\operatorname{Cat}(2)=2, \\
\operatorname{Cat}^{\prime \prime \prime}(5 / 3) & =\operatorname{Cat}^{\prime \prime}(3 / 2)=\operatorname{Cat}^{\prime}(2)=\operatorname{Cat}(1)=1 .
\end{aligned}
$$

The process stabilizes because Cat $^{\prime}(1)=1$.

\section{Rational Dyck Paths}

At the heart of our constructions lies a family of lattice paths called rational Dyck paths. A rational Dyck path is a path from $(0,0)$ to $(b, a)$ in the integer lattice $\mathbb{Z}^{2}$ using steps of the form $(1,0)$ and $(0,1)$ and staying above the diagonal $y=\frac{a}{b} x$. (Because $a$ and $b$ are coprime, it will never touch the diagonal.) More specifically, we call this an $x$-Dyck path or an $(a, b)$-Dyck path. For example, Figure 1 displays a $(5,8)$-Dyck path. When $a$ and $b$ are clear from context, we will sometimes refer to $(a, b)$-Dyck paths as simply Dyck paths.

Note that the final step of an $(n, n+1)$-Dyck path must travel from $(n, n)$ to $(n, n+1)$. Upon removing this step we obtain a path from $(0,0)$ to $(n, n)$ that stays weakly above the line of slope 1 ; that is, we obtain a classical Dyck path. The following result generalizes the fact that there are Cat $(n, n+1)$ classical Dyck paths, can be proven using the Cycle Lemma of Dvorestky and Motzkin [DM], and is perhaps best attributed to 'folklore'.

Theorem 1 For $a \neq b$ coprime positive integers, the number of $(a, b)$-Dyck paths is the Catalan number $\operatorname{Cat}(a, b)=\frac{1}{a+b}\left(\begin{array}{c}a+b \\ a, b\end{array}\right)$.

The following refinement from [ALW] can also be proven using the Cycle Lemma.

Theorem 2 The number of $(a, b)$-Dyck paths with i nontrivial vertical runs is the Narayana number

$$
\operatorname{Nar}(a, b ; i):=\frac{1}{a}\left(\begin{array}{c}
a \\
i
\end{array}\right)\left(\begin{array}{c}
b-1 \\
i-1
\end{array}\right)
$$


and the number of $(a, b)$-Dyck paths with $r_{j}$ vertical runs of length $j$ is the Kreweras number

$$
\operatorname{Krew}(a, b ; \mathbf{r}):=\frac{1}{b}\left(\begin{array}{c}
b \\
r_{0}, r_{1}, \ldots, r_{a}
\end{array}\right)=\frac{(b-1) !}{r_{0} ! r_{1} ! \cdots r_{a} !} .
$$

Equivalently, the first formula counts the $(a, b)$-Dyck paths with $i-1$ valleys. We include trivial vertical runs of length 0 in the second formula for aesthetic reasons. For example, the path in Figure 1 has 3 nontrivial vertical runs (i.e. 2 valleys) and $\mathbf{r}=(5,1,2,0,0,0)$. The rational Narayana numbers will appear below as the $h$-vector of the rational associahedron.

\section{Rational Associahedra}

\subsection{Simplicial Complexes}

We recall a collection of definitions related to simplicial complexes. A simplicial complex $\Delta$ on a finite ground set $E$ is a collection of subsets of $E$ such that if $S \in \Delta$ and $T \subseteq S$, then $T \in \Delta$. The elements of $\Delta$ are called faces, the maximal elements of $\Delta$ are called facets, and $\Delta$ is called pure if all of its facets have the same cardinality. The dimension of a face $S \in \Delta$ is $\operatorname{dim}(S):=|S|-1$ and the dimension of $\Delta$ is the maximum dimension of a face in $\Delta$. Observe that the 'empty face' $\emptyset$ has dimension -1 .

If $\Delta$ is a $d$-dimensional simplicial complex, the $f$-vector of $\Delta$ is the integer sequence $f(\Delta)=\left(f_{-1}, f_{0}, \ldots, f_{d}\right)$, where $f_{-1}=1$ and $f_{i}$ is the number of $i$-dimensional faces in $\Delta$ for $0 \leq$ $i \leq d$. The reduced Euler characteristic $\chi(\Delta)$ is given by $\chi(\Delta):=\sum_{i=-1}^{d}(-1)^{i} f_{i}$. The $h$-vector of $\Delta$ is the sequence $h(\Delta)=\left(h_{-1}, h_{0}, \ldots, h_{d}\right)$ defined by the following polynomial equation in $t$ : $\sum_{i=-1}^{d} f_{i}(t-1)^{d-i}=\sum_{k=-1}^{d} h_{k} t^{k}$. The sequences $f(\Delta)$ and $h(\Delta)$ determine one another completely for any simplicial complex $\Delta$.

Shellability is a key property possessed by some pure simplicial complexes which determines the homotopy type and $h$-vector of the complex. Let $\Delta$ be a pure $d$-dimensional simplicial complex. A total order $F_{1} \prec \ldots \prec F_{r}$ on the facets $F_{1}, \ldots, F_{r}$ of $\Delta$ is called a shelling order if for $2 \leq k \leq r$, the subcomplex of the simplex $F_{k}$ defined by $C_{k}:=\left(\bigcup_{i=1}^{k-1} F_{i}\right) \cap F_{k}$ is a pure $(d-1)$-dimensional simplicial complex. The complex $\Delta$ is called shellable if there exists a shelling order on its facets; it can be shown that any pure $d$-dimensional shellable simplicial complex is homotopy equivalent to a wedge of spheres, all of dimension $d$.

The number of the spheres in the homotopy type of a shellable complex can be read off from the shelling order. More precisely, let $\Delta$ be a pure $d$-dimensional simplicial complex which is shellable and let $F_{1} \prec \cdots \prec F_{r}$ be a shelling order on its facets. For $1 \leq k \leq r$, there exists a unique minimal face $M_{k}$ of the simplex $F_{k}$ which is not contained in the union $\bigcup_{i=1}^{k-1} F_{i}$ of the facets which appear earlier in the shelling order. The multiset of dimensions $\left\{\operatorname{dim}\left(M_{1}\right), \operatorname{dim}\left(M_{2}\right), \ldots, \operatorname{dim}\left(M_{k}\right)\right\}$ of these minimal added faces is independent of the shelling order. In fact, we have that $i^{\text {th }}$ entry $h_{i}$ of the $h$-vector $h(\Delta)$ equals the number of minimal faces $M_{k}$ with $\operatorname{dim}\left(M_{k}\right)=i$. Moreover, the complex $\Delta$ is homotopy equivalent to a wedge of $h_{d}$ copies of the $d$-dimensional sphere. For future use, we recall the well-known fact that adding a unique minimal face at each stage characterizes shelling orders.

Lemma 3 Let $\Delta$ be a pure simplicial complex and let $F_{1} \prec \cdots \prec F_{r}$ be a total order on the facets of $\Delta$. The order $\prec$ is a shelling order if and only if for $1 \leq k \leq r$ there exists a unique minimal face $M_{k}$ of the simplex $F_{k}$ which is not contained in $\bigcup_{i=1}^{k-1} F_{i}$. 


\subsection{Construction, Basic Facts, and Conjectures}

For $n \geq 3$, let $\mathbb{P}_{n}$ denote the regular $n$-gon. Recall that the (dual of the) classical associahedron $\operatorname{Ass}(n, n+$ 1)consists of all (noncrossing) collections of diagonals of $\mathbb{P}_{n+2}$-the dissections of $\mathbb{P}_{n+2}$-ordered by inclusion. The diagonals of $\mathbb{P}_{n+2}$ are therefore the vertices of $\operatorname{Ass}(n, n+1)$ and the facets of $\operatorname{Ass}(n, n+1)$ are labeled by the maximal dissections-the triangulations of $\mathbb{P}_{n+2}$. Associahedra were introduced by Stasheff $[\mathbf{S t}]$ in the context of nonassociative products arising in algebraic topology. Since its introduction, the associahedron has become one of the most well-studied complexes in geometric combinatorics, with connections to the permutohedron and exchange graphs of cluster algebras.

The classical associahedron has a Fuss analog defined as follows. Let $m \geq 1$ be a Fuss parameter. The Fuss associahedron $\operatorname{Ass}(n, m n+1)$ has as its facets the collection of all dissections of $\mathbb{P}_{m n+2}$ into $(m+2)$-gons. Fuss associahedra arise in the study of the generalized cluster complexes of Fomin and Reading.

We define our further generalization $\operatorname{Ass}(a, b)$ of the classical associahedron as follows. The vertices of Ass $(a, b)$ will correspond to certain diagonals in $\mathbb{P}_{b+1}$ and the faces will correspond to certain dissections of $\mathbb{P}_{b+1}$. Label the vertices of $\mathbb{P}_{b+1}$ clockwise with $1,2, \ldots, b+1$.

Given any Dyck path $D$ and any lattice point $P=(i, j)$ which is the bottom of a north step in $D$, we associate a diagonal $e(P)$ in $\mathbb{P}_{b+2}$ as follows. Consider the line $\ell$ with equation $(y-j)=\frac{a}{b}(x-i)$. This line intersects the path $D$ in the lattice point $P$ and in at least one other point to the right of $P$. Let $Q$ be the leftmost such point to the right of $P$ and let $(r, s)$ be the coordinates of $Q$. By coprimality and the fact that $b>a$, we have that $i+1<s<b$ and $s$ is not an integer. Let $e(P)$ be the diagonal $(i,\lceil s\rceil)$ in $\mathbb{P}_{b+1}$, where $\lceil s\rceil$ is the smallest integer $\geq s$. Define a subset $F(D)$ of diagonals of $\mathbb{P}_{b+1}$ by

$$
F(D):=\{e(P): P \text { is the bottom of a north step in } D\} .
$$

The right of Figure 2 shows the collection $F(D)$ of diagonals corresponding to the given Dyck path $D$ on $\mathbb{P}_{9}$. It is topologically clear that the collection $F(D)$ of diagonals in $\mathbb{P}_{b+1}$ is noncrossing for any Dyck path $D$. The sets $F(D)$ form the facets of our simplicial complex.

Definition 4 For $a<b$, the simplicial complex $\operatorname{Ass}(a, b)$ has as its ground set the collection of diagonals of $\mathbb{P}_{b+1}$ and facets $\{F(D): D$ is an $(a, b)$-Dyck path $\}$.

The following basic facts about $\operatorname{Ass}(a, b)$ can be proven directly from its definition.

Proposition 5 1. The simplicial complex $\operatorname{Ass}(a, b)$ is pure and has dimension $a-2$.

2. The number of facets in $\operatorname{Ass}(a, b)$ is $\operatorname{Cat}(a, b)$.

3. Define a subset $S(a, b)$ of $[b-1]$ by $S(a, b)=\left\{\left\lfloor\frac{i b}{a}\right\rfloor: 1 \leq i<a\right\}$, where $\lfloor s\rfloor$ is the greatest integer $\leq s$. A diagonal of $\mathbb{P}_{b+1}$ which separates $i$ vertices from $b-i-1$ vertices appears as a vertex in the complex $\operatorname{Ass}(a, b)$ if and only if $i \in S(a, b)$.

We will call a diagonal $e$ of $\mathbb{P}_{b+1}$ which satisfies the hypothesis in Part 3 of Proposition $5(a, b)$ admissible. The vertex set of $\operatorname{Ass}(a, b)$ consists precisely of the $(a, b)$-admissible diagonals in $\mathbb{P}_{b+1}$.

Proof: Part 1 follows from the fact that an $(a, b)$-Dyck path contains $a$ north steps. For Part 2, observe that if $D$ and $D^{\prime}$ are distinct Dyck paths, the multisets of $x$-coordinates of the bottoms of the north steps of $D$ and $D^{\prime}$ are distinct. In particular, this means that $F(D)$ and $F\left(D^{\prime}\right)$ are distinct sets of diagonals in 


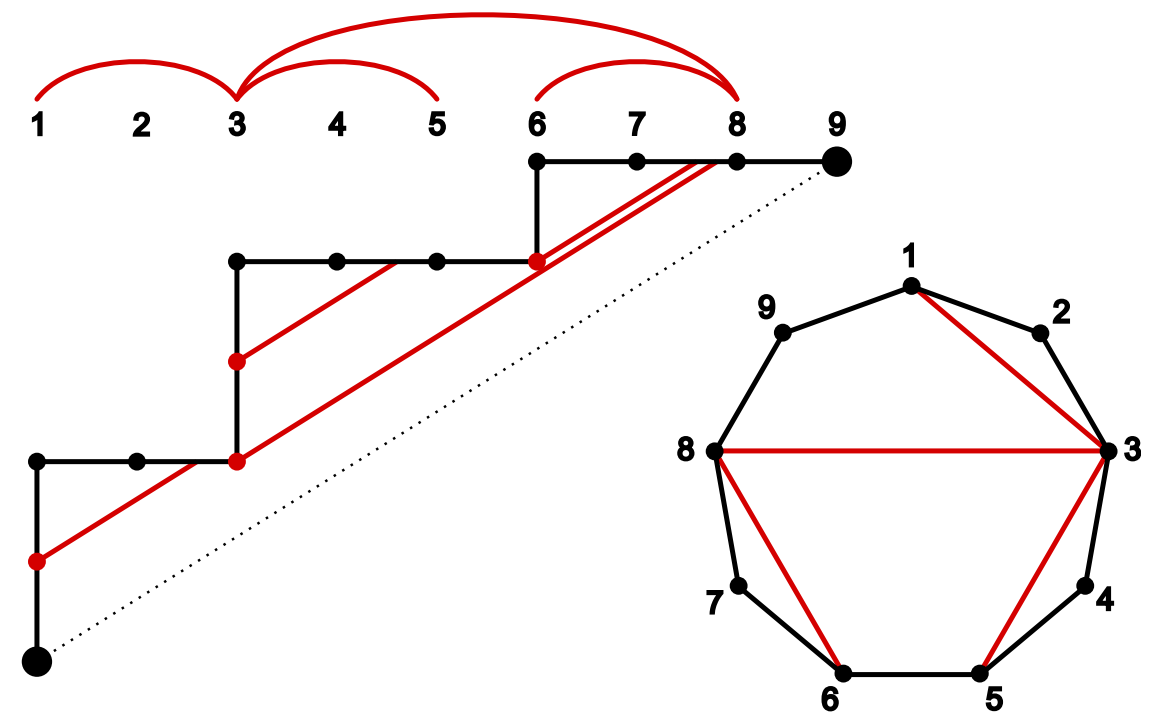

Fig. 2: A $(5,8)$-Dyck path and the corresponding dissection of $\mathbb{P}_{9}$.

$\mathbb{P}_{b+1}$. Part 2 follows from the fact that there are Cat $(a, b)$ Dyck paths. Part 3 is a geometric observation about lines of slope $\frac{a}{b}$.

In the classical case $b=a+1$ and the Fuss case $b=k a+1$, the faces of the associahedron $\operatorname{Ass}(a, b)$ are given by collections of $(a, b)$-admissible diagonals in $\mathbb{P}_{b+1}$ which are mutually noncrossing. Given this characterization, it is clear that the associahedron carries an action of the cyclic group $\mathbb{Z}_{b+1}$ given by rotation in these cases. Neither of these statements remains true at the rational level of generality. Indeed, when $(a, b)=(3,5)$, the diagonals $(1,5)$ and $(3,5)$ of $\mathbb{P}_{6}$ are 3,5 -admissible and mutually noncrossing. However, the set $\{(1,5),(3,5)\}$ is not a face of $\operatorname{Ass}(3,5)$. It can also be checked that $\operatorname{Ass}(3,5)$ is not closed under rotation of $\mathbb{P}_{6}$.

In spite of the last paragraph, we conjecture that $\operatorname{Ass}(a, b)$ carries a rotation action 'up to homotopy'. More precisely, let $\operatorname{Ass}^{\prime}(a, b)$ denote the simplicial complex whose faces are collections of mutually noncrossing $(a, b)$-admissible diagonals in $\mathbb{P}_{b+1}$. It is clear that $\operatorname{Ass}^{\prime}(a, b)$ carries a rotation action and that $\operatorname{Ass}(a, b)$ is a subcomplex of $\operatorname{Ass}^{\prime}(a, b)$.

Before stating our conjecture, we recall what it means for a complex to collapse onto a subcomplex; this is a combinatorial deformation retraction. Let $\Delta$ be a simplicial complex, $F \in \Delta$ be a facet, and suppose $F^{\prime} \subset F$ satisfies $\left|F^{\prime}\right|=|F|-1$. If $F^{\prime}$ is not contained in any facet of $\Delta$ besides $F$, we can perform an elementary collapse by replacing $\Delta$ with $\Delta-\left\{F, F^{\prime}\right\}$. A simplicial complex is said to collapse onto a subcomplex if the subcomplex can be obtained by a sequence of elementary collapses.

Conjecture 6 The complexes $\operatorname{Ass}(a, b)$ and $\operatorname{Ass}^{\prime}(a, b)$ are homotopy equivalent. In fact, the complex $\operatorname{Ass}^{\prime}(a, b)$ collapses onto the subcomplex $\operatorname{Ass}(a, b)$.

Figure 3 displays $\operatorname{Ass}(2,5)$ (shown in blue) and $\operatorname{Ass}(3,5)$ (shown in red) as subcomplexes of the sphere Ass $(4,5)$. The complex $\operatorname{Ass}^{\prime}(2,5)$ coincides with $\operatorname{Ass}(2,5)$ and the complex $\operatorname{Ass}^{\prime}(3,5)$ is obtained from 


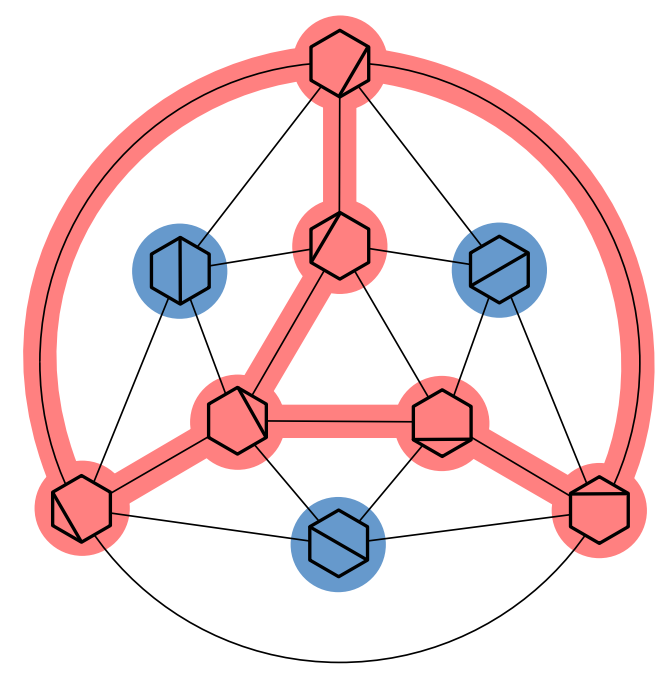

Fig. 3: $\operatorname{Ass}(2,5)$ and $\operatorname{Ass}(3,5)$ are Alexander dual within $\operatorname{Ass}(4,5)$.

the complex $\operatorname{Ass}(3,5)$ by adding the middle and exterior triangles to the red complex. Observe that Ass $(3,5)$ can be obtained by performing two elementary collapses on Ass' $(3,5)$.

Conjecture 6 would also have implications regarding Alexander duality. Recall that two topological subspaces $X$ and $Y$ of a fixed sphere $S$ are said to be Alexander dual to one another if $Y$ is homotopy equivalent to the complement of $X$ in $S$. With $b>1$ fixed, we have that $a$ and $b$ are coprime for $1 \leq a<b$ if and only if $b-a$ and $b$ are coprime. Both of the complexes $\operatorname{Ass}(a, b)$ and $\operatorname{Ass}(a-b, b)$ sit within the classical associahedron $\operatorname{Ass}(b-1, b)$. The proof of Conjecture 6 would imply that $\operatorname{Ass}(a, b)$ and $\operatorname{Ass}(a-b, b)$ are Alexander dual.

Proposition 7 Let $a<b$ be coprime for $b>1$. The subcomplexes $\operatorname{Ass}(a, b)$ and $\operatorname{Ass}(b-a, b)$ are Alexander dual within the sphere $\operatorname{Ass}(b-1, b)$. If Conjecture 6 is true, then the subcomplexes $\operatorname{Ass}(a, b)$ and $\operatorname{Ass}(b-a, b)$ are also Alexander dual within $\operatorname{Ass}(b-1, b)$.

Proof: It is routine to check that any diagonal of $\mathbb{P}_{b+1}$ is either $(a, b)$-admissible or $((b-a), b)$-admissible, but not both. This means that the vertex sets of $\operatorname{Ass}^{\prime}(a, b)$ and $\operatorname{Ass}^{\prime}(b-a, b)$ partition the vertex set of the simplicial sphere $\operatorname{Ass}^{\prime}(b-1, b)$. By definition, the faces of $\operatorname{Ass}^{\prime}(a, b)$ and $\operatorname{Ass}^{\prime}(b-a, b)$ are precisely the faces of $\operatorname{Ass}(b-1, b)$ whose vertex sets are contained in $\operatorname{Ass}^{\prime}(a, b)$ and $\operatorname{Ass}^{\prime}(b-a, b)$, respectively. It follows that the complement of $\operatorname{Ass}^{\prime}(a, b)$ inside $\operatorname{Ass}(b-1, b)$ deformation retracts onto $\operatorname{Ass}^{\prime}(b-a, b)$. This proves the first statement. The second statement is clear.

\subsection{Shellability and $f$ - and $h$-vectors}

We will prove that the simplicial complex $\operatorname{Ass}(a, b)$ is shellable by giving an explicit shelling order on its facets. This shelling order will be induced by lexicographic order on the partitions whose Ferrers diagrams lie to the northwest of $(a, b)$-Dyck paths. 
Theorem 8 The simplicial complex $\operatorname{Ass}(a, b)$ is shellable, hence homotopy equivalent to a wedge of spheres. Moreover, there is a total order $D_{1} \prec D_{2} \prec \cdots \prec D_{\mathrm{Cat}(a, b)}$ on the set of $(a, b)$-Dyck paths which induces a shelling order on the facets of $\operatorname{Ass}(a, b)$ such that the dimension of the minimal face added upon addition of the facet $F\left(D_{i}\right)$ equals the number of nonempty vertical runs in $D_{i}$, less one.

Proof: (Sketch.) We will find it convenient to identify the facets of $\operatorname{Ass}(a, b)$ with both Dyck paths and partitions. We define a family of concepts which will be used for this proof only.

A partition $\lambda$ is a weakly decreasing sequence $\left(\lambda_{1} \geq \cdots \geq \lambda_{k}>0\right)$ of positive integers. The Ferrers diagram associated with $\lambda$ consists of $\lambda_{i}$ left justified boxes in row $i$ (we are using English notation). We will use the lexicographic total order $\prec$ on partitions defined by $\lambda \prec \mu$ if there exists an $i \geq 1$ such that $\lambda_{j}=\mu_{j}$ for $1 \leq j<i$ and $\lambda_{i}<\mu_{i}$. (We append an infinite string of zeros to the ends of $\lambda$ and $\mu$, if necessary, for these relations to make sense.)

Given any $(a, b)$-Dyck path $D$, let $\lambda(D)$ be the partition whose Ferrers diagram consists of the lattice boxes to the northwest of $D$ in the rectangle with corners $(0,0)$ and $(b, a)$. For example, if $(a, b)=(5,8)$ and $D$ is the path in Figure 2 then $\lambda(D)=(5,2,2)$. It is clear that distinct Dyck paths give rise to distinct partitions, so the facets of $\operatorname{Ass}(a, b)$ are labeled by either $(a, b)$-Dyck paths or by partitions $\lambda$ which satisfy $\lambda_{i} \leq \max \left(\left\lfloor\frac{(a-i) b}{a}\right\rfloor, 0\right)$ for all $i$.

Let $\lambda^{(1)} \prec \cdots \prec \lambda^{(\operatorname{Cat}(a, b))}$ be the restriction of lexicographic order to set of partitions which satisfy $\lambda_{i} \leq \max \left(\left\lfloor\frac{(a-i) b}{a}\right\rfloor, 0\right)$ for all $i$. In particular, we have that $\lambda^{(1)}$ is the empty partition and $\lambda_{i}^{(\mathrm{Cat}(a, b))}=$ $\max \left(\left\lfloor\frac{(a-i) b}{a}\right\rfloor, 0\right)$. The total order $\prec$ induces a total order $D_{1} \prec \cdots \prec D_{\text {Cat }(a, b)}$ on $(a, b)$-Dyck paths and a total order $F\left(D_{1}\right) \prec \cdots \prec F\left(D_{\mathrm{Cat}(a, b)}\right)$ on the facets of $\operatorname{Ass}(a, b)$.

In the case $(a, b)=(3,5)$, our order on partitions is

$$
(0,0) \prec(1,0) \prec(1,1) \prec(2,0) \prec(2,1) \prec(3,0) \prec(3,1) .
$$

The corresponding order on facets of $\operatorname{Ass}(3,5)$ (written as diagonal sets in $\mathbb{P}_{6}$ ) is

$$
\begin{aligned}
& \{(1,3),(1,5)\} \prec\{(2,4),(1,5)\} \prec\{(2,4),(2,6)\} \prec \\
& \{(1,3),(3,5)\} \prec\{(2,6),(3,5)\} \prec\{(1,3),(4,6)\} \prec\{(2,4),(4,6)\} .
\end{aligned}
$$

We will prove that $\prec$ is a shelling order on the facets of $\operatorname{Ass}(a, b)$ and that the minimal added faces corresponding to $\prec$ have the required dimensions. In fact, we will be able to describe these minimal added faces explicitly. Given any Dyck path $D$, recall that the corresponding facet $F(D)$ in $\operatorname{Ass}(a, b)$ is given by $F(D)=\{e(P): P$ is the bottom of a north step in $D\}$. We define the valley face $V(D)$ to be the subset of $F(D)$ given by $V(D):=\{e(P): P$ is a valley in $D\}$.

Claim: $1 \leq k \leq \operatorname{Cat}(a, b)$, the valley face $V\left(D_{k}\right)$ is the unique minimal face of $F\left(D_{k}\right)$ which is not contained in $\bigcup_{i=1}^{k-1} F\left(D_{i}\right)$.

By Lemma 3 and the discussion preceding it, this claim implies that $\prec$ is a shelling order with the dimension of the minimal added face as described (observe that in any Dyck path, the number of valleys equals the number of vertical runs), completing the proof of the theorem. The proof of this claim is omitted in this extended abstract.

As a corollary to the above result, we get product formulas for the $f$ - and $h$-vectors of $\operatorname{Ass}(a, b)$, as well as its reduced Euler characteristic. Define the rational Kirkman numbers by

$$
\operatorname{Kirk}(a, b ; i):=\frac{1}{a}\left(\begin{array}{c}
a \\
i
\end{array}\right)\left(\begin{array}{c}
b+i-1 \\
i-1
\end{array}\right) .
$$


Corollary 9 Let $\left(f_{-1}, f_{0}, \ldots, f_{a-2}\right)$ and $\left(h_{-1}, h_{0}, \ldots, h_{a-2}\right)$ be the $f$ - and $h$-vectors of $\operatorname{Ass}(a, b)$. For $1 \leq i \leq a$ we have that $f_{i-1}=\operatorname{Kirk}(a, b ; i)$ and $h_{i-1}=\operatorname{Nar}(a, b ; i)$. The reduced Euler characteristic of $\operatorname{Ass}(a, b)$ is the derived Catalan number $\operatorname{Cat}^{\prime}(a, b)$.

Proof: By Theorem 8 and Lemma 3, we have that $h_{i-1}$ equals the number of $(a, b)$-Dyck paths which have exactly $i$ vertical runs. By Theorem 2 this equals the Narayana number $\operatorname{Nar}(a, b ; i)$.

To prove the statement about the $f$-vector, one must check that

$$
\sum_{i=-1}^{a-2} \operatorname{Kirk}(a, b ; i+1)(t-1)^{a-i-2}=\sum_{k=1}^{a-2} \operatorname{Nar}(a, b ; k+1) t^{k} .
$$

The statement about the Euler characteristic reduces to proving that

$$
\sum_{i=-1}^{a-2}(-1)^{i+1} \operatorname{Kirk}(a, b ; i+1)=\operatorname{Cat}^{\prime}(a, b) .
$$

Both of these identities are left to the reader.

Conjecture 6 and Proposition 7 assert that the symmetry $(a<b) \leftrightarrow(b-a<b)$ on pairs of coprime positive integers shows up in rational associahedra as an instance of Alexander duality. Corollary 9 shows that the categorification Cat $(x) \mapsto \operatorname{Cat}^{\prime}(x)$ of the Euclidean algorithm presented in Section 2 sends the number of facets of $\operatorname{Ass}(a, b)$ to the reduced Euler characteristic of Ass $(a, b)$. This 'categorifies' the number theoretic properties of rational Catalan numbers to the context of associahedra.

\section{Acknowledgements}

The authors would like to thank Susanna Fishel, Stephen Griffeth, Jim Haglund, Mark Haiman, Brant Jones, Nick Loehr, Vic Reiner, Dennis Stanton, Hugh Thomas, Monica Vazirani, and Greg Warrington for helpful discussions.

B. Rhoades was partially supported by NSF grant DMS - 1068861.

\section{References}

[Arm] D. Armstrong, Generalized noncrossing partitions and combinatorics of Coxeter groups, Mem. Amer. Math. Soc., 202 (949).

[ALW] D. Armstrong, N. Loehr, and G. Warrington, Rational parking functions. In preparation, 2012.

[Ath1] C. A. Athanasiadis, Characteristic polynomials of subspace arrangements and finite fields, $A d v$. Math., 122 (1996), 193-233.

[Ath2] C. A. Athanasiadis, On a refinement of the generalized Catalan numbers for Weyl groups, Trans. Amer. Math. Soc., 357 (1) (2004), 179-196.

[Cay] A. Cayley, A theorem on trees, Quart. J. Math. 23 (1889), 376-378. 
[CP] P. Cellini and P. Papi, Ad-nilpotent ideals of a Borel subalgebra II, J. Algebra 258 (1) (2002), 112 121.

[DM] A. Dvoretsky and T.H. Motzkin, A problem of arrangements, Duke Math. J. 14 (1947), 305-313.

[Edel] P. Edelman, Chain enumeration and noncrossing partitions, Discrete Math. 31 (2) (1980), 171180.

[FV] S. Fishel and M. Vazirani, A bijection between (bounded) dominant Shi regions and core partitions, DMTCS Proceedings, 22nd International Conference on Formal Power Series and Algebraic Combinatorics, (FPSAC 2010).

[FR] S. Fomin and N. Reading, Generalized cluster complexes and Coxeter combinatorics, Int. Math. Res. Notices, 44 (2005), 2709-2757.

[FZ] S. Fomin and A. Zelevinsky, Cluster algebras II: Finite type classification, Inventiones Math., 154 (2003), 63-121.

[GH] A.M. Garsia and M. Haiman, A remarkable $q, t$-Catalan sequence and $q$-Lagrange inversion, $J$. Algebraic Combin. 5 (1996), no. 3, 191-244.

[Gord] I. Gordon, On the quotient ring by diagonal invariants, Invent. Math. 153 (2003), 503-518.

[Hai1] M. Haiman, Conjectures on the quotient ring by diagonal invariants, J. Algebraic Combin. 3 (1994) $17-76$.

[Hai2] M. Haiman, Hilbert schemes, polygraphs and the Macdonald positivity conjecture. J. Amer. Math. Soc. 14 (4) (2001), 941-1006.

[Kirk] T.P. Kirkman, On the k-partitions of the r-gon and r-ace, Phil. Trans. Royal Soc. London, 147 (1857), 217-272.

[Krew] G. Kreweras, Sur les partitions non crois/'ees d'un cycle, Discrete Math. 1 (1972), 333-350.

[Nar] T.V. Narayana, Sur les trellis formés par les partitions d'un entier et leurs applications à la théories des probabilités, C. R. Acad. Sci. Paris, 240 (1955), 1188-1189.

[Rei] V. Reiner, Non-crossing partitions for classical reflection groups, Discrete Math. 177 (1-3) (1997), 195-222.

[Shi1] J.-Y. Shi, Sign types corresponding to an affine Weyl group, J. London Math. Soc., 35 (1987), 56-74.

[Som] E. Sommers, B-stable ideals in the nilradical of a Borel subalgebra, Canad. Math. Bull. 48 (2005), $460-472$.

[Stan] R. Stanley, Catalan addendum. http://www-math.mit.edu/ rstan/ec/catadd.pdf

[St] J. D. Stasheff, Homotopy associativity of H-spaces. I, II. Trans. Amer. Math. Soc. 108 (1963), 275292; ibid. 293-312.

[W] H. Weyl, The classical groups, Princeton University Press, (1939). 